\title{
Does lumbar drainage reduce postoperative cerebrospinal fluid leak after endoscopic endonasal skull base surgery? A prospective, randomized controlled trial
}

\author{
Nathan T. Zwagerman, MD, ${ }^{1}$ Eric W. Wang, MD, ${ }^{2}$ Samuel S. Shin, MD, PhD, ${ }^{1}$ Yue-Fang Chang, PhD, ${ }^{1}$ \\ Juan C. Fernandez-Miranda, MD, ${ }^{1}$ Carl H. Snyderman, MD, MBA, ${ }^{2}$ and Paul A. Gardner, MD1 \\ Departments of ${ }^{1}$ Neurological Surgery and ${ }^{2}$ Otolaryngology, University of Pittsburgh School of Medicine, Pittsburgh, \\ Pennsylvania
}

\begin{abstract}
OBJECTIVE Based on a null hypothesis that the use of short-term lumbar drainage (LD) after endoscopic endonasal surgery (EES) for intradural pathology does not prevent postoperative CSF leaks, a trial was conducted to assess the effect of postoperative LD on postoperative CSF leak following standard reconstruction.
\end{abstract}

METHODS A prospective, randomized controlled trial of lumbar drain placement after endoscopic endonasal skull base surgery was performed from February 2011 to March 2015. All patients had 3-month follow-up data. Surgeons were blinded to which patients would or would not receive the drain until after closure was completed. An a priori power analysis calculation assuming $80 \%$ of power, $5 \%$ postoperative CSF leak rate in the no-LD group, and $16 \%$ in the LD group determined a planned sample size of 186 patients. A routine data and safety check was performed with every 50 patients being recruited to ensure the efficacy of randomization and safety. These interim tests were run by a statistician who was not blinded to the arms they were evaluating. This study accrued 230 consecutive adult patients with skull base pathology who were eligible for endoscopic endonasal resection. Inclusion criteria (high-flow leak) were dural defect greater than $1 \mathrm{~cm}^{2}$ (mandatory), extensive arachnoid dissection, and/or dissection into a ventricle or cistern. Sixty patients were excluded because they did not meet the inclusion criteria. One hundred seventy patients were randomized to either receive or not receive a lumbar drain.

RESULTS One hundred seventy patients were randomized, with a mean age of 51.6 years (range 19-86 years) and $38 \%$ were male. The mean BMl for the entire cohort was $28.1 \mathrm{~kg} / \mathrm{m}^{2}$. The experimental cohort with postoperative LD had an $8.2 \%$ rate of CSF leak compared to a $21.2 \%$ rate in the control group (odds ratio 3.0, 95\% confidence interval 1.2-7.6, $p=0.017)$. In 106 patients in whom defect size was measured intraoperatively, a larger defect was associated with postoperative CSF leak (6.2 vs $2.9 \mathrm{~cm}^{2}, p=0.03$ ). No significant difference was identified in BMI between those with (mean $\left.28.4 \pm 4.3 \mathrm{~kg} / \mathrm{m}^{2}\right)$ and without (mean $\left.28.1 \pm 5.6 \mathrm{~kg} / \mathrm{m}^{2}\right)$ postoperative CSF leak $(p=0.79)$. Furthermore, when patients were grouped based on BMl $<25,25-29.9$, and $>30 \mathrm{~kg} / \mathrm{m}^{2}$, no difference was noted in the rates of CSF fistula $(p=$ 0.97).

CONCLUSIONS Among patients undergoing intradural EES judged to be at high risk for CSF leak as defined by the study's inclusion criteria, perioperative LD used in the context of vascularized nasoseptal flap closure significantly reduced the rate of postoperative CSF leaks.

Clinical trial registration no.: NCT03163134 (clinicaltrials.gov).

https://thejns.org/doi/abs/10.3171/2018.4.JNS172447

KEYWORDS lumbar drain; CSF leak; endoscopic endonasal approach; nasoseptal flap; pituitary surgery; skull base

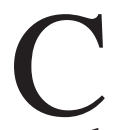
EREBROSPINAL fluid leak after skull base surgery can have serious sequelae. Prevention includes reconstruction with multiple layers and often includes vascularized tissue. To lower intracranial pressure and thus potentially prevent postoperative CSF leaks, lumbar drainage (LD) is often used in the perioperative and postoperative period. ${ }^{1,4}$ With the increasing utilization of endoscopic endonasal surgery (EES), reducing postoperative CSF leak is paramount as this remains a major limitation of the approach, with rates cited as high as $40 \% .{ }^{8}$ Drains,

ABBREVIATIONS CI = confidence interval; DVT = deep vein thrombosis; $E E S$ = endoscopic endonasal surgery; LD = lumbar drainage; OR = odds ratio; PE = pulmonary emboli.

SUBMITTED October 17, 2017. ACCEPTED April 17, 2018.

INCLUDE WHEN CITING Published online October 19, 2018; DOI: 10.3171/2018.4.JNS172447. 
however, have significant potential for side effects, including spinal headache, radiculopathy, overdrainage (with risk of hemorrhage), and decreased patient mobilization.

The utility of LD for CSF leaks has been reported, 3,6 but there is a lack of evidence of efficacy of LD after EES. Variations in repair techniques and substrates make comparisons difficult. ${ }^{22,23}$ The nasoseptal flap has become universally accepted as a primary reconstructive method for high-flow leaks, ${ }^{17,40}$ but there is little consensus on the use of LD, the criteria for placing lumbar drains, or the protocol for lumbar drain maintenance..$^{1,9,33}$

The best evidence available is class $\mathrm{III}^{2}$ and this lack of evidence complicates efforts to both guide and standardize postoperative care. This study was a meta-analysis evaluating the use of LD for CSF leaks and found that there was insufficient data to support the use of a lumbar drain. In order to provide a higher level of evidence, a randomized controlled trial was conducted to assess the effect of postoperative LD on postoperative CSF leak following standard (vascularized) reconstruction in the setting of high-flow defects.

\section{Methods}

\section{Study Design and Participants}

All adult patients undergoing EES at the Center for Cranial Base Surgery of the University of Pittsburgh Medical Center from February 2011 to March 2015 were evaluated for enrollment. Inclusion criteria were a dural defect greater than $1 \mathrm{~cm}^{2}$ (mandatory), extensive arachnoid dissection, and/or dissection into a ventricle or cistern. Entry criteria were determined using previous cases of patients with known high-flow CSF leaks. Dural defects greater than 1 $\mathrm{cm}^{2}$ were mandatory to catch all patients with high-flow leaks. This was determined by the surgeon's approximation using a 0.5 -inch cottonoid in the period before defects were measured with a metric ruler. Based on the surgical approach, a patient could not have met criteria 2 or 3 without criteria 1 being met. That is, no patient in this series underwent arachnoid dissection or third ventricle entry without an opening large enough for bimanual dissection and thus $>1 \mathrm{~cm}^{2}$. Patients under the age of 18 , those undergoing an open or combined surgery (extracranial pericranial flap reconstruction was not considered open or combined), or those who refused consent were excluded from the study. An a priori power analysis calculation assuming $80 \%$ of power, $5 \%$ postoperative CSF leak rate in the LD group, and $16 \%$ in the no-LD group determined a planned sample size of 186 patients. A routine data and safety check was performed with every 50 patients being recruited to ensure the efficacy of randomization and safety (significant benefit or harm). The O'Brien-Fleming method was used to determine the boundaries of critical values of the interim tests. ${ }^{29}$ The alpha level for the first interim test (50 patients) was 0.00016 , for the second interim test (100 patients) it was 0.0075 , and for the third interim test (150 patients) it was 0.029 . These interim tests were run by a statistician who was not blinded to the arms they were evaluating and were also performed without revealing the results to the investigators or any clinician involved, as long as they did not meet significance. If a patient who was enrolled but found to be ineligible after repair was identified, the sealed envelope was used for the next enrolled patient.

This study was approved by the University of Pittsburgh IRB and written consent was obtained for each patient participating in the study. There was no funding provided for this trial. Departmental and Cranial Base Center salary support is provided for the statistician and research coordinator who were involved. This study was registered with the ClinicalTrials.gov database (https://clinicaltrials. gov), and its registration no. is NCT03163134.

\section{Randomization and Blinding}

Eligible patients were randomized to either LD at 10 $\mathrm{ml} /$ hour for 72 hours or no drainage. Binary block (10-patient) randomization was performed using a random number generator in Microsoft Excel (2010) for the entire sample size prior to the initiation of the trial. A blinded and sealed sequentially numbered envelope was provided on the day of surgery by an investigator or coordinator not directly involved with the surgery. A high-flow intraoperative CSF leak ${ }^{40}$ was confirmed in all patients with the above inclusion criteria. At the completion of surgery (after the skull base defect was completely repaired) a sealed envelope containing instructions for either "drain" or "no drain" was opened by the operating room nursing staff.

\section{Intervention}

Reconstruction was performed by an otolaryngologist and a neurosurgeon using a "standard" reconstruction protocol, including intradural collagen as an inlay graft and a vascularized flap. ${ }^{16,19,32}$ Antibiotic prophylaxis was given for 48 hours in the form of intravenous ceftriaxone, after which the regimen switched to oral ceftin for 5 days. The antibiotics were the same in both arms and were based on nasal packing, not lumbar drain utilization. In cases in which the septal flap was not available, an extracranial pericranial flap $(n=2)$ or lateral nasal wall flap $(n=7)$ was used. ${ }^{34}$ Multilayer reconstruction (3-4 layers) including fascia lata and/or fat graft for large posterior or anterior (fascia only) fossa defects was applied in some cases later in the series. As noted, the entirety of the surgery, including flap harvesting, tumor resection, and skull base reconstruction, was performed while blinded to the randomization. When indicated, the lumbar drain was then placed immediately after surgery and prior to emergence from anesthesia. In rare cases in which a drain could not be placed in the operating room, it was placed at the earliest possible time by interventional radiology (typically the following morning). After placement of a lumbar drain but prior to opening it, a noncontrasted head CT scan was performed to evaluate for intracranial pathology including hemorrhage or excessive pneumocephalus. After the lumbar drain was opened, instructions for the nursing staff included titration to drain $10 \mathrm{ml}$ of CSF every hour. The drains were in place for approximately 72 hours. If the drain stopped working, it was evaluated, flushed, and replaced if needed.

\section{Outcome Measures}

The primary outcome measure was the presence of postoperative CSF leak during the follow-up period (30 days). 


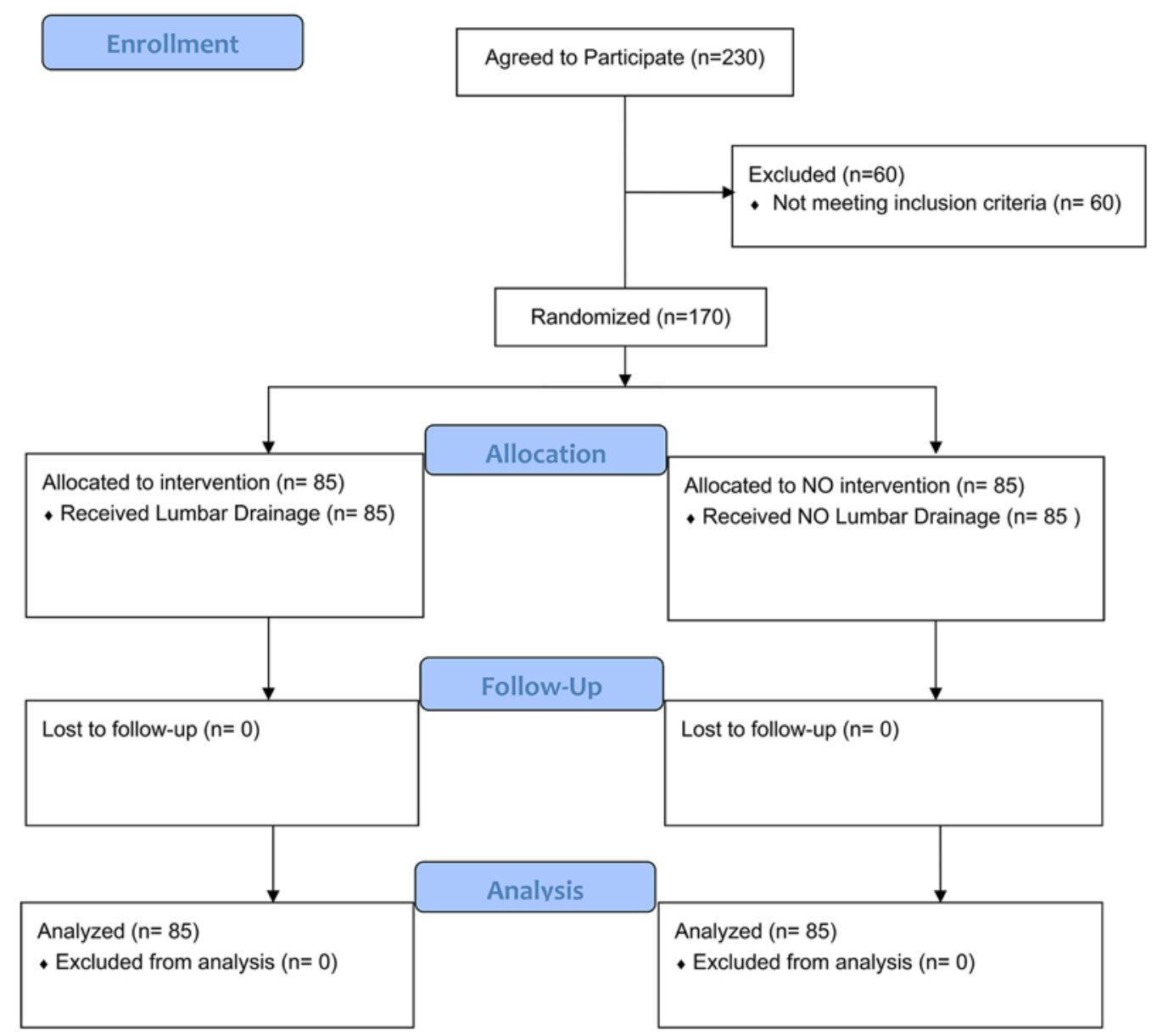

FIG. 1. Randomization arms and flow chart. Figure is available in color online only.

The presence of a CSF leak was confirmed by either operative exploration and/or biochemical (beta-2 transferrin) testing. Demographic variables such as age, sex, BMI, prior surgery, as well as surgical-specific variables such as tumor location, tumor type, and defect size were recorded for analysis. Precise measurement of the defect size was added as a secondary measure after the initial 62 patients. At the time of the operation, defect size was measured intraoperatively prior to any reconstruction using a trimmed plastic ruler and captured with the endoscopic recording system.

\section{Statistical Analysis}

Data were analyzed using SAS software (SAS Institute). Chi-square and Fisher's exact tests were used for the categorical variables (e.g., CSF leak, tumor location) comparisons while the t-test and Wilcoxon rank-sum test were used for the continuous variables (e.g., age, BMI, tumor size) comparisons. Logistic regression models were applied to assess the multivariate association between CSF leak and other factors. Statistical significance was determined to be at $p<0.05$.

\section{Results}

This study was terminated at 170 patients after a rou- tine data safety check found a clear and significant difference in CSF leak rate between the experimental (LD, $8.2 \%$ ) and control (no LD, 21.2\%) groups (odds ratio [OR] $3.0,95 \%$ confidence interval $[\mathrm{CI}] 1.2-7.6, \mathrm{p}=0.017$ ). A randomization flow chart with enrollment is given in Fig. 1. All enrolled patients completed the study. Mean age at time of surgery was 51.6 years (range 19-86 years) with $38 \%$ being male patients. The mean BMI for the entire cohort was $28.1 \mathrm{~kg} / \mathrm{m}^{2}$. Demographic information for the two groups is shown in Table 1. The patients were divided evenly between the experimental $(\mathrm{LD}, \mathrm{n}=85)$ and control (no LD, $\mathrm{n}=85$ ) groups. There were no significant differences between the groups in age, BMI, or tumor location. Of the 170 cases, 2 patients were reconstructed with a pericranial flap and 7 patients had lateral nasal wall flaps. The most significant predictor of a postoperative CSF leak was the lack of a lumbar drain ( $\mathrm{p}=0.017)$.

There was no difference in age (49.2 \pm 15.4 vs $52.1 \pm$ 15.3 years, $\mathrm{p}=0.38)$ or BMI $(28.4 \pm 4.3$ vs $28.1 \pm 5.6 \mathrm{~kg} /$ $\mathrm{m}^{2}, \mathrm{p}=0.79$ ) when comparing patients with and without CSF leak. Patients were subdivided into groups based on BMI: those with a BMI $<25,25-29.9$, and $>30 \mathrm{~kg} / \mathrm{m}^{2}$ each had a CSF leak rate of approximately $14 \%$ with no difference between these groups (OR 1.4, 95\% CI 0.6-3.4, $\mathrm{p}=0.97$ ). Additionally, 56 patients who had previous sur- 
TABLE 1. Demographic information within the LD and no-LD groups

\begin{tabular}{lccc}
\hline \multicolumn{1}{c}{ Variable } & LD $(\mathrm{n}=85)$ & No LD $(\mathrm{n}=85)$ & $\mathrm{p}$ Value \\
\hline Mean age $\pm \mathrm{SD}, \mathrm{yrs}$ & $51.0 \pm 15.1$ & $52.3 \pm 15.5$ & 0.572 \\
\hline Females, $\mathrm{n}(\%)$ & $56(65.9)$ & $49(57.7)$ & 0.269 \\
\hline Mean BMI $\pm \mathrm{SD}, \mathrm{kg} / \mathrm{m}^{2}$ & $27.6 \pm 5.6$ & $28.7 \pm 5.2$ & 0.178 \\
\hline Pathology & & & \\
\hline Anterior, $\mathrm{n}(\%)$ & $18(21.2)$ & $17(20.0)$ & \\
\hline Posterior, $\mathrm{n}(\%)$ & $24(28.2)$ & $26(30.6)$ & \\
\hline Suprasellar, $\mathrm{n}(\%)$ & $43(50.6)$ & $42(49.4)$ & 0.942 \\
\hline CSF leaks, $\mathrm{n}(\%)$ & $7(8.2)$ & $18(21.2)$ & 0.017 \\
\hline
\end{tabular}

gery (EES/transcranial) were not more likely to have a CSF leak ( $p=0.43)$. In 19 patients who had prior EES, there was no significantly increased risk of CSF leak ( $p$ $=0.49$ ). Of those who had previous EES, 7 had lateral nasal wall flaps for reconstruction and the rest had revision nasoseptal flaps. Overall, lateral nasal wall flaps showed a trend toward postoperative CSF leak compared to nasoseptal flap repairs $(\mathrm{p}=0.18)$.

Logistic regression analysis indicated that those without LD were 2.9 times more likely to have a CSF leak (OR $2.9,95 \%$ CI 1.1-7.4, $\mathrm{p}=0.029)$ than those with LD when controlling for age $(p=0.28)$, sex $(p=0.31)$, BMI $(p=$ $0.90)$, and any previous surgery $(\mathrm{p}=0.67)$.

In patients who had LDs, there were 9 with deep vein thromboses (DVTs) and pulmonary emboli (PE) compared to 5 patients in the control group $(p=0.26)$. There were also no differences in postoperative respiratory complications $(\mathrm{p}=1.0)$, meningitis, or any infection with LD $(\mathrm{p}=0.81$ and $\mathrm{p}=0.58$, respectively).

There were 3 direct lumbar drain complications (3.5\%): 2 patients suffered from postoperative spinal headaches and required a lumbar blood patch, and 1 patient had a retained catheter that was observed without consequence. There were no long-term symptoms of radiculopathy. No patient suffered from intracranial hypotension causing a hematoma or tension pneumocephalus.

A post hoc secondary analysis of the location of the dural defect and its effect on postoperative CSF leak rates was completed (Table 2). Patients with suprasellar tumors had a decreased risk of postoperative CSF leak compared to patients with tumors of the anterior or posterior fossa ( $7 \%$ vs $20 \%$ vs $22 \%$ leak, respectively; $\mathrm{p}=0.019$ ) using the chi-square test to compare all 3 groups. This difference remained significant for patients in the control group ( $9.5 \%$ vs $35 \%$ vs $31 \%, \mathrm{p}=0.032)$, but was not observed in the LD cohort ( $4.7 \%$ vs $11 \%$ vs $13 \%, \mathrm{p}=0.50)$ in prespecified subgroup analyses.

The anterior fossa pathology consisted of 35 patients who had either meningiomas of the olfactory groove or anterior planum sphenoidale, or esthesioneuroblastomas. Within this group, the CSF leak rate in the LD patients was $11.1 \%$ in comparison to $35.3 \%$ in the control group $(p=0.12)$. All 7 of the CSF leaks in this group were from patients who harbored meningiomas.

Of the 50 patients with posterior fossa pathology, chor-
TABLE 2. Tumor pathology based on location, number of patients harboring the pathology, and number of CSF leaks (in brackets)

\begin{tabular}{lll}
\hline \multicolumn{1}{c}{ Anterior } & \multicolumn{1}{c}{ Posterior } & \multicolumn{1}{c}{ Suprasellar } \\
\hline $\begin{array}{l}\text { Meningioma 28 [7] } \\
\begin{array}{l}\text { Esthesioneuro- } \\
\text { blastoma } 7\end{array}\end{array}$ & Meningioma 16 [3] & Adenomama 20 [3] \\
\hline & Epidermoid 2 [1] & Craniopharyngioma 18 \\
\hline & Chondroblastoma 1 & Rathke's cleft cyst 6 [1] \\
\hline & Chondrosarcoma 1 [1] & Aneurysm 3 [1] \\
\hline & Schwannoma 1 & Epidermoid 1 [1] \\
\hline Meningoencephalocele 1 & Dermoid 1 \\
\hline & Aneurysm 1 & Lymphoid 1 \\
\hline & Cavernous malforma- \\
& & tion 1 \\
\hline & & Germ cell 1 \\
\hline
\end{tabular}

Totals: anterior, 35 patients, 7 leaks (20\%); posterior, 50 patients, 11 leaks (22\%); and suprasellar, 84 patients, 6 leaks $(7 \%)$.

domas and meningiomas were the primary tumor types. Within this group, the CSF leak rate for those with a drain was $12.5 \%$ compared to $30.8 \%$ without $(p=0.12)$. There was no significant difference in CSF leak rates between the two pathologies $(p=0.9)$.

Finally, 85 of the patients had suprasellar pathology consisting of adenomas with suprasellar invasion, craniopharyngiomas, and tuberculum sellae meningiomas. Among these patients, there was no significant difference in CSF leak rates between the LD (4.7\%) and control $(9.5 \%)$ groups $(p=0.43)$.

The average dural defect size in 25 patients from the anterior fossa group in whom it was measured was 7.2 $\mathrm{cm}^{2}$, in 22 patients with posterior fossa pathology it was $3.8 \mathrm{~cm}^{2}$, and in 59 patients with suprasellar tumors it was $1.6 \mathrm{~cm}^{2}$. Further evaluation of the impact of defect size on CSF leak found a strong trend, with the average defect in those with a leak being $6.2 \mathrm{~cm}^{2}$ compared to $2.9 \mathrm{~cm}^{2}$ in those without a leak $(p=0.03)$.

Using the patients with tumor size and location data, we applied a logistic regression model to assess the effects of tumor location and tumor size on the CSF leak. Using a stepwise selection procedure in the logistic regression analysis we found that only defect size was significantly associated with CSF leak (OR 1.86, 95\% CI 1.05-3.32, p = 0.035).

\section{Discussion}

CSF leak remains one of the major challenges of EES. ${ }^{8,9,25,39}$ Vascularized tissue has become the foundation for reconstruction. ${ }^{27,28}$ Horiguchi et al. compared patients who underwent EES with nasoseptal flap reconstruction and those who underwent EES with fat graft or fascia lata reconstruction and found there was a significant difference in CSF leak rates (9.5\% vs $27.3 \%$, respectively). ${ }^{18}$ This is in agreement with other studies that reported low CSF leak rates with nasoseptal flap reconstruction ranging from $5.7 \%$ to $0 \%$ leak rate..$^{10,11}$ As a result, current 
EES for most commonly performed procedures provides a much lower risk of CSF leak than previously described. ${ }^{14,15}$ Based on potential risks for serious complications of LD, the low rate of CSF leak with current reconstruction techniques, and the wide variation in its application, the necessity of LD after EES remained undefined.

LD is used frequently following many skull base surgeries to prevent CSF leaks. In retrospective studies for transcranial approaches, ${ }^{7}$ the rates of postoperative CSF leakage were $35 \%$ in patients with no perioperative lumbar drain and $12 \%$ in patients with LD. Another study that included CSF fistulae from spinal and cranial surgeries as well as traumatic causes ${ }^{37}$ showed the rate of CSF leak at $6 \%$ when a lumbar drain is used, without investigation of CSF leak rates when a lumbar drain is not used. However, there have been few prospective studies assessing the usefulness of LD in skull base surgery, and none regarding EES. ${ }^{1,74}$ An important confounder is identifying CSF leaks as high flow or low flow. A practical definition of "high-flow CSF leak" is one that violates a ventricle or cistern and requires a more robust reconstruction, ${ }^{12,30}$ which was the primary inclusion criterion for this study, i.e., a large dural defect defined as $1 \mathrm{~cm}^{2}$.

Clinical practice varies widely and there are two recent studies that suggest that LD does not need to be routinely used since the advent of the nasoseptal flap by Hadad et al. ${ }^{16}$ Garcia-Navarro et al. noted that there was no association between lumbar drain usage and postoperative CSF leak. Although they did describe grade 3 leaks with suprasellar or transclival defects and noted that these patients had a higher risk of CSF leak (12\%) in their series, most of their cases were suprasellar with $86 \%$ of the surgeries using transtuberculum/transplanum approaches. These authors continued to advise perioperative LD in these patients. ${ }^{13}$ Eloy et al. retrospectively reviewed 59 patients who underwent endoscopic repair of high-flow CSF leaks with a nasoseptal flap but without LD; none had a postoperative CSF leak. ${ }^{11}$ Again, the majority (42 patients) had suprasellar or sellar pathology, with only 14 with anterior cranial fossa lesions and 3 with clival defects. They noted that 57 patients had a defect $>1 \mathrm{~cm}^{2}$. Bakhsheshian et al. found that several studies evaluating LD in the endoscopic endonasal repair of CSF leaks indicated no clear benefit of LD. ${ }^{5}$ However, they made no mention of the size or location of the defects and none of these studies were randomized. A recent review of LD usage in endoscopic skull base surgery concluded that there is much variation regarding $\mathrm{LD}$ and there is no definitive protocol. ${ }^{38}$

It is noteworthy that the CSF leak rate in our study was higher than our expected CSF leak rate of 5\%. At the time of development, published CSF leak rates for endoscopic endonasal skull base surgery was variable depending on factors such as location, defect size, grade of leak, closure technique, and pathology. Our predetermined CSF leak rate was determined based on the best published data to adequately answer the question on the benefit of lumbar drain usage. However, this literature includes all types of pathologies and many grades (low flow and high flow) of CSF leak. Our study intentionally focused on the highest grades of CSF leak to emphasize the impact and also be consistent with current best practices based on prior stud- ies, which suggested that lumbar drains are not needed for low-flow leaks.

This study provides class I data to address this unanswered question. The inclusion criteria selected for defects and locations that are most susceptible to a postoperative CSF leak and did not include any situations that would dilute the denominator (low-flow defects/adenomas, no intraoperative leak). Bias was minimized by not notifying the surgeons of the randomization of the patient until after closure was completed. Drains were placed at the end of surgery, as this is our standard procedure. The study was stopped early due to clear efficacy of LD in decreasing postoperative CSF.

Secondary analysis showed that defect location and size contributed to leakage rates. For anterior and posterior fossa defects, LD clearly resulted in lower postoperative CSF leaks. This was not true, however, for the suprasellar cohort (e.g., craniopharyngiomas with third ventricle extension, suprasellar pituitary adenomas, or tuberculum meningiomas). Interestingly, in our series, there were no leaks from patients who had a suprasellar meningioma or a craniopharyngioma only from adenomas with significant arachnoid invasion/dissection. It is possible that a much smaller difference in CSF leak rates for the suprasellar group was not detected by this study due to sample size; however, this group was by far the largest $(n=84)$. While this may be a result of small sample size, based on the potential difference, we would need to assign 465 patients per group for a total of 930 suprasellar tumors to possibly demonstrate a statistical difference. This value reflects the lack of significance and would never realistically be accrued. Indeed, the lack of significance for suprasellar defects is consistent with the majority of the literature, consisting of nonrandomized series, largely composed of sellar or suprasellar tumors that did not demonstrate a need for LD. $1,5,8,13,24$

Dural defect size was measured intraoperatively in 106 patients but was not routinely performed early in our series. We found a strong trend toward significance between size and leak rate $(p=0.03)$. However, this was confounded by a clear size difference between the anterior/ posterior defects and the suprasellar defects. Using defect size and location as predictors, we found that defect size played a larger and significant role in postoperative CSF leaks $(\mathrm{p}=0.035)$. One explanation is that a standard $(20$ $\mathrm{cm}^{2}$ ) or even extended nasoseptal flap $\left(28 \mathrm{~cm}^{2}\right)$ provides ample coverage of a suprasellar defect but may be inadequate for larger defects. ${ }^{31}$ In addition, the rotation of a nasoseptal flap is ideal for a suprasellar defect, which lies in the middle of the "sweet spot" for this flap, whereas clival or cribriform defects extend to the caudal and rostral limits of its reach.

LD has various associated risks that must be balanced against its benefit. Common complaints include headache, nausea, and vomiting, which occurs in 13\%-63\% of cases. ${ }^{1,36}$ Meningitis and other infections have been reported in $4 \%-10 \%$ of cases ${ }^{21}$ Continuation of LD for more than several days postoperatively is risky because a longer duration of LD is correlated with higher rates of infection..$^{36}$ Other rare but serious complications include neurological deficits due to excessive drainage with tonsillar herniation, 
acute or delayed intracranial hypotension, ${ }^{20,35}$ intracranial venous thrombosis, ${ }^{26}$ lumbar nerve root irritation, pneumocephalus, and retained catheters. These are all in addition to the systemic complications associated with the relative immobilization of the patient required for safe $\mathrm{LD}$ usage.

Our study indicates no significant association between LD and postoperative infection or meningitis. Likely this is a result of the relatively short duration of LD and the adherence to aseptic techniques when manipulating drains. The duration of drainage (72 hours) was specifically chosen to limit the risk of infection. Because these patients are less mobile, we evaluated for DVTs, PE, and respiratory complications and found no significant difference between the two groups. In all patients, subcutaneous heparin was begun on postoperative day 2 while the lumbar drain was in place. This is, of course, dependent on other practices such as aggressive mobilization of patients regardless of $\mathrm{LD}$, early prophylactic administration of subcutaneous heparin (48 hours postoperatively), and the quality of nursing care.

It is important to note that 2 of our $\mathrm{LD}$ patients required a postoperative blood patch for spinal headaches. Although both resolved with this relatively simple treatment, there was associated morbidity as these patients suffer from debilitating headaches and the inability to ambulate, which may lead to other complications. In addition, these are only the most intractable cases, and similar, milder symptoms are present in many patients. There was also 1 patient who suffered from a retained lumbar catheter that was severed during placement. In addition to the medical risks of LD, there is a significant economic cost associated with LD that is offset by the economic benefits of preventing a CSF leak. The low rates of drain-associated morbidity appear to be offset by the advantages of drainage in anterior and posterior fossa defects.

As a result, 72 hours of postoperative $\mathrm{LD}$ at $10 \mathrm{ml} /$ hour for patients with large anterior or posterior fossa intradural defects is recommended. However, in small defects, especially in the suprasellar region, vascularized reconstruction may be sufficient.

A significant limitation to this study is the fact that it only uses data from a single institution and a single closure technique (multilayer closure). In this situation, LD decreases postoperative CSF leaks. As endoscopic skull base surgery techniques improve and CSF leak rates lower even further, LD may no longer become as significant. We also understand that different closure techniques, especially for limited defects, may produce different results and therefore may not have the same results with LD. Regardless, this paper does present level 1 evidence that lumbar drains reduce postoperative CSF leaks in patients with sizable dural defects.

\section{Conclusions}

LD after EES reduces postoperative CSF leaks. This appears especially true in the setting of large dural defects associated with anterior and posterior fossa pathologies. LD for suprasellar defects is not recommended unless there are other risk factors for CSF leak.

\section{References}

1. Ackerman PD, Spencer DA, Prabhu VC: The efficacy and safety of preoperative lumbar drain placement in anterior skull base surgery. J Neurol Surg Rep 74:1-9, 2013

2. Ahmed OH, Marcus S, Tauber JR, Wang B, Fang Y, Lebowitz RA: Efficacy of perioperative lumbar drainage following endonasal endoscopic cerebrospinal fluid leak repair: a metaanalysis. Otolaryngol Head Neck Surg 156:52-60, 2017

3. Albu S, Florian IS, Bolboaca SD: The benefit of early lumbar drain insertion in reducing the length of CSF leak in traumatic rhinorrhea. Clin Neurol Neurosurg 142:43-47, 2016

4. Allen KP, Isaacson B, Purcell P, Kutz JW Jr, Roland PS: Lumbar subarachnoid drainage in cerebrospinal fluid leaks after lateral skull base surgery. Otol Neurotol 32:1522-1524, 2011

5. Bakhsheshian J, Hwang MS, Friedman M: What is the evidence for postoperative lumbar drains in endoscopic repair of CSF leaks? Laryngoscope 125:2245-2246, 2015

6. Bell RB, Dierks EJ, Homer L, Potter BE: Management of cerebrospinal fluid leak associated with craniomaxillofacial trauma. J Oral Maxillofac Surg 62:676-684, 2004

7. Bien AG, Bowdino B, Moore G, Leibrock L: Utilization of preoperative cerebrospinal fluid drain in skull base surgery. Skull Base 17:133-139, 2007

8. Borg A, Kirkman MA, Choi D: endoscopic endonasal anterior skull base surgery: a systematic review of complications during the past 65 years. World Neurosurg 95:383-391, 2016

9. Dehdashti AR, Stofko D, Okun J, Obourn C, Kennedy T: Endoscopic endonasal reconstruction of skull base: repair protocol. J Neurol Surg B Skull Base 77:271-278, 2016

10. El-Sayed IH, Roediger FC, Goldberg AN, Parsa AT, McDermott MW: Endoscopic reconstruction of skull base defects with the nasal septal flap. Skull Base 18:385-394, 2008

11. Eloy JA, Kuperan AB, Choudhry OJ, Harirchian S, Liu JK: Efficacy of the pedicled nasoseptal flap without cerebrospinal fluid (CSF) diversion for repair of skull base defects: incidence of postoperative CSF leaks. Int Forum Allergy Rhinol 2:397-401, 2012

12. Esposito F, Dusick JR, Fatemi N, Kelly DF: Graded repair of cranial base defects and cerebrospinal fluid leaks in transsphenoidal surgery. Neurosurgery 60 (4 Suppl 2):295-304, 2007

13. Garcia-Navarro V, Anand VK, Schwartz TH: Gasket seal closure for extended endonasal endoscopic skull base surgery: efficacy in a large case series. World Neurosurg 80:563-568, 2013

14. Gardner PA, Kassam AB, Snyderman CH, Carrau RL, Mintz AH, Grahovac S, et al: Outcomes following endoscopic, expanded endonasal resection of suprasellar craniopharyngiomas: a case series. J Neurosurg 109:6-16, 2008

15. Gardner PA, Kassam AB, Thomas A, Snyderman CH, Carrau RL, Mintz AH, et al: Endoscopic endonasal resection of anterior cranial base meningiomas. Neurosurgery 63:36-54, 2008

16. Hadad G, Bassagasteguy L, Carrau RL, Mataza JC, Kassam A, Snyderman CH, et al: A novel reconstructive technique after endoscopic expanded endonasal approaches: vascular pedicle nasoseptal flap. Laryngoscope 116:1882-1886, 2006

17. Harvey RJ, Parmar P, Sacks R, Zanation AM: Endoscopic skull base reconstruction of large dural defects: a systematic review of published evidence. Laryngoscope 122:452-459, 2012

18. Horiguchi K, Murai H, Hasegawa Y, Hanazawa T, Yamakami I, Saeki N: Endoscopic endonasal skull base reconstruction using a nasal septal flap: surgical results and comparison with previous reconstructions. Neurosurg Rev 33:235-241, 2010

19. Kassam AB, Thomas A, Carrau RL, Snyderman CH, Vescan A, Prevedello D, et al: Endoscopic reconstruction of the cra- 
nial base using a pedicled nasoseptal flap. Neurosurgery 63 (1 Suppl 1):ONS44-ONS53, 2008

20. Kim YS, Kim SH, Jung SH, Kim TS, Joo SP: Brain stem herniation secondary to cerebrospinal fluid drainage in ruptured aneurysm surgery: a case report. Springerplus 5:247, 2016

21. Kitchel SH, Eismont FJ, Green BA: Closed subarachnoid drainage for management of cerebrospinal fluid leakage after an operation on the spine. J Bone Joint Surg Am 71:984987, 1989

22. Leng LZ, Brown S, Anand VK, Schwartz TH: "Gasket-seal" watertight closure in minimal-access endoscopic cranial base surgery. Neurosurgery 62 (5 Suppl 2):ONSE342-ONSE343, 2008

23. Luginbuhl AJ, Campbell PG, Evans J, Rosen M: Endoscopic repair of high-flow cranial base defects using a bilayer button. Laryngoscope 120:876-880, 2010

24. Mascarenhas L, Moshel YA, Bayad F, Szentirmai O, Salek AA, Leng LZ, et al: The transplanum transtuberculum approaches for suprasellar and sellar-suprasellar lesions: avoidance of cerebrospinal fluid leak and lessons learned. World Neurosurg 82:186-195, 2014

25. McCoul ED, Anand VK, Singh A, Nyquist GG, Schaberg MR, Schwartz TH: Long-term effectiveness of a reconstructive protocol using the nasoseptal flap after endoscopic skull base surgery. World Neurosurg 81:136-143, 2014

26. Miglis MG, Levine DN: Intracranial venous thrombosis after placement of a lumbar drain. Neurocrit Care 12:83-87, 2010

27. Moyer JS, Chepeha DB, Teknos TN: Contemporary skull base reconstruction. Curr Opin Otolaryngol Head Neck Surg 12:294-299, 2004

28. Nameki H, Kato T, Nameki I, Ajimi Y: Selective reconstructive options for the anterior skull base. Int J Clin Oncol 10:223-228, 2005

29. O'Brien PC, Fleming TR: A multiple testing procedure for clinical trials. Biometrics 35:549-556, 1979

30. Patel MR, Stadler ME, Snyderman CH, Carrau RL, Kassam AB, Germanwala AV, et al: How to choose? Endoscopic skull base reconstructive options and limitations. Skull Base 20:397-404, 2010

31. Peris-Celda M, Pinheiro-Neto CD, Funaki T, FernandezMiranda JC, Gardner P, Snyderman C, et al: The extended nasoseptal flap for skull base reconstruction of the clival region: an anatomical and radiological study. J Neurol Surg B Skull Base 74:369-385, 2013

32. Prevedello DM, Thomas A, Gardner P, Snyderman CH, Carrau RL, Kassam AB: Endoscopic endonasal resection of a synchronous pituitary adenoma and a tuberculum sellae meningioma: technical case report. Neurosurgery 60 (4 Suppl 2): $\mathrm{E} 401,2007$

33. Ransom ER, Palmer JN, Kennedy DW, Chiu AG: Assessing risk/benefit of lumbar drain use for endoscopic skull-base surgery. Int Forum Allergy Rhinol 1:173-177, 2011

34. Rivera-Serrano CM, Bassagaisteguy LH, Hadad G, Carrau RL, Kelly D, Prevedello DM, et al: Posterior pedicle lateral nasal wall flap: new reconstructive technique for large defects of the skull base. Am J Rhinol Allergy 25:e212-e216, 2011

35. Samadani U, Huang JH, Baranov D, Zager EL, Grady MS: Intracranial hypotension after intraoperative lumbar cerebrospinal fluid drainage. Neurosurgery 52:148-152, 2003
36. Scheithauer S, Bürgel U, Bickenbach J, Häfner H, Haase G, Waitschies B, et al: External ventricular and lumbar drainage-associated meningoventriculitis: prospective analysis of time-dependent infection rates and risk factor analysis. Infection 38:205-209, 2010

37. Shapiro SA, Scully T: Closed continuous drainage of cerebrospinal fluid via a lumbar subarachnoid catheter for treatment or prevention of cranial/spinal cerebrospinal fluid fistula. Neurosurgery 30:241-245, 1992

38. Stokken J, Recinos PF, Woodard T, Sindwani R: The utility of lumbar drains in modern endoscopic skull base surgery. Curr Opin Otolaryngol Head Neck Surg 23:78-82, 2015

39. Thorp BD, Sreenath SB, Ebert CS, Zanation AM: Endoscopic skull base reconstruction: a review and clinical case series of 152 vascularized flaps used for surgical skull base defects in the setting of intraoperative cerebrospinal fluid leak. Neurosurg Focus 37(4):E4, 2014

40. Zanation AM, Carrau RL, Snyderman CH, Germanwala AV, Gardner PA, Prevedello DM, et al: Nasoseptal flap reconstruction of high flow intraoperative cerebral spinal fluid leaks during endoscopic skull base surgery. Am J Rhinol Allergy 23:518-521, 2009

41. Zhan R, Chen S, Xu S, Liu JK, Li X: Postoperative low-flow cerebrospinal fluid leak of endoscopic endonasal transsphenoidal surgery for pituitary adenoma-wait and see, or lumbar drain? J Craniofac Surg 26:1261-1264, 2015

\section{Disclosures}

The authors report no conflict of interest concerning the materials or methods used in this study or the findings specified in this paper.

\section{Author Contributions}

Conception and design: Gardner, Wang, Fernandez-Miranda, Snyderman. Acquisition of data: Gardner, Wang, Shin, FernandezMiranda, Snyderman. Analysis and interpretation of data: Zwagerman, Shin, Chang. Drafting the article: Zwagerman, Shin Critically revising the article: Gardner, Zwagerman, Wang, Shin, Fernandez-Miranda, Snyderman. Reviewed submitted version of manuscript: all authors. Approved the final version of the manuscript on behalf of all authors: Gardner. Statistical analysis: Zwagerman, Chang. Administrative/technical/material support: Gardner, Zwagerman, Wang, Shin, Fernandez-Miranda, Snyderman. Study supervision: Gardner, Wang, Fernandez-Miranda, Snyderman.

\section{Supplemental Information \\ Previous Presentations}

Portions of this work were presented in abstract form at the 2016 Annual Meeting of the AANS in Chicago, Illinois, April 30-May 4.

\section{Correspondence}

Paul A. Gardner: UPMC Center for Cranial Base Surgery, Pittsburgh, PA.gardpa@upmc.edu. 